\title{
Heavy Flavor Wilson Coefficients in Deep-Inelastic Scattering: Recent Results*
}

\section{J. Ablinger}

Research Institute for Symbolic Computation (RISC), Johannes Kepler University, Altenbergerstraße 69, A-4040, Linz, Austria

\section{A. Behring,}

Institut für Theoretische Teilchenphysik und Kosmologie, RWTH Aachen University, Sommerfeldstr. 16, D-52074 Aachen, Germany

\section{J. Blümlein; A. De Freitas}

Deutsches Elektronen-Synchrotron, DESY, Platanenallee 6, D-15738 Zeuthen, Germany

\section{A. von Manteuffel}

Department of Physics and Astronomy, Michigan State University, East Lansing, MI 48824, USA

\section{Schneider}

Research Institute for Symbolic Computation (RISC), Johannes Kepler University,

Altenbergerstraße 69, A-4040, Linz, Austria

We present recent analytic results for the 3-loop corrections to the massive operator matrix element $A_{Q g}^{(3)}$ for further color factors. These results have been obtained using the method of arbitrarily large moments. We also give an overview on the results which were obtained solving all difference and differential equations for the corresponding master integrals that factorize at first order.

QCDEV2017, JLAB, Newport News, VA, USA,

May 22-26, 2017

*This work was supported in part by the Austrian Science Fund (FWF) grant SFB F50 (F5009-N15), the European Commission through contract PITN-GA-2012-316704 (HIGGSTOOLS), and National Science Foundation under Grant No. 1719863.

†Speaker 


\section{Introduction}

The precision determination of the strong coupling constant $\alpha_{s}\left(M_{Z}^{2}\right)$ [1], of the parton distribution functions [2], and of the heavy quark masses $m_{c}$ and $m_{b}$ [3] from the world deep inelastic data requires both the knowledge of the NNLO massless and massive QCD corrections to the parton densities. Theses quantities are fundamental parameters of the Standard Model or serve as an important input for other measurements at hadron colliders to determine further fundamental parameters, like those of the top-quark [4] and the Higgs sectors [5]. These are important places at present to find deviations from the Standard Model and therefore need the most precise possible description.

During the last years, essential progress has been made in calculating the massive 3-loop corrections to the deep-inelastic structure functions [6-21]. Different computational methods have been worked out in Refs. [22-24]. Progress has also been made in the 2-mass case, where several OMEs have been calculated, cf. [25-28]. For a recent summary on this see Ref. [29].

All but one of the massive operator matrix elements (OMEs) have been calculated. In this note we describe recent developments of the project concerning the calculation of the $\operatorname{OME} A_{Q g}^{(3)}$, as well as some technical details of the calculation, the present results, and challenges for its completion.

The paper is organized as follows. In Section 2 we describe the method of arbitrarily large moments and calculation techniques for first order factorizing differential and difference equations. The contributions to $A_{Q g}^{(3)}$ that have been obtained already by using these methods are described in Section 3. There we also give an outline on the methods to be used to calculate the remaining terms, which are related to new functions, the iterative non-iterative integrals [30]. Section 4 contains the conclusions.

\section{The method of arbitrarily large moments and calculation techniques for first order factorizing equations}

In the calculation of the massive OMEs, we reduce the Feynman diagrams to master integrals using integration by parts (IBP) relations [31]. Depending on their complexity, different calculation methods exist to compute these integrals. The simplest integrals can be expressed in terms of (generalized) hypergeometric functions [32]. In more general cases, one seeks Mellin-Barnes representations [33] and uses the residue theorem to obtain convergent infinite sum representations. Such sums are also obtained in the case of the representation through hypergeometric functions after expanding in the dimensional parameter $\varepsilon$. In Mellin $N$ space, also sum representations are obtained. For finite integrals, one may also use the method of hyperlogarithms [23,34-36]. Having full control on the IBP reduction, one may map divergent integrals into convergent ones [37]. Another powerful tool consists in the method of differential equations, cf. Refs. [38] and [24]. In the present case, they can be obtained from the IBP relations by differentiating w.r.t. the resummation parameter $x$ for the operator insertions, cf. [14]. Inserting the formal power series in $x$ into the differential equations, one obtains recurrence relations, which, as well as the summation problems mentioned before, can be solved by difference field and ring methods [39-47] using the algorithms implemented in the packages Sigma [48,49], EvaluateMultiSums and SumProduction [50]. In various places the package HarmonicSums [51-55] is used to operate on special functions of 
different kinds emerging throughout the calculation. In some cases we also use multi-integration according to the Almkvist-Zeilberger theorem [56] as an integration method. This method is implemented in the package MultiIntegrate [51]. All these methods have been described in detail in Ref. [24]. In all cases where difference equations factorize at first order, Sigma will find the solution in Mellin $N$ space. The transition to $x$-space is possible applying algorithms implemented in the package HarmonicSums.

As it is well known, massive single-scale problems starting at 3-loop order are partly related to master integrals for which the corresponding difference and differential equations do not factorize in first order, [30]. Yet cases in which this applies to the master integrals, but not to their sum projected on some color- $\zeta$ factors, i.e. terms which can be distinguished by the corresponding product of Casimir operators and potential (multiple) zeta values [57], may be dealt with. Considering fixed moments $N$, cf. also [7], the results are given by rational numbers and multiple zeta values only [57]. The appearance of new higher functions like iterated non-iterative integrals manifests only in the corresponding rational sequences but not in new special numbers. These sequences can now be analyzed and the difference equations which describe them can be determined $[58,59]$ provided enough Mellin moments can be calculated. This is possible using the algorithm [60], implemented in the package SolveCoupledSystem, that can compute arbitrarily large moments. The corresponding difference equations can now be analyzed by the package Sigma, which will determine all its 1st order factors, and in the present case, a remainder term not factorizing in first order. We will illustrate this in the case of the unrenormalized OME $\hat{\hat{A}}_{Q g}^{(3)}$ in Section 3.

\section{The contributions to $\mathrm{A}_{\mathrm{Qg}}^{(3)}$ through first order factorizing equations}

In the following we calculate 3-loop contributions to the massive OME $A_{Q g}^{(3)}$ in the single heavy quark mass case. The 1358 Feynman diagrams are generated using QGRAF [61] and are brought into a form in which the operator insertions [7] are resummed into a propagator, cf. [14], using a formal resummation parameter $x$. The color algebra is performed using Color [62]. We use Reduze2 [63] ${ }^{1}$ to map the problem to 340 master integrals. 224 master integrals were calculated using the methods described in [24]. We derive differential equations for all master integrals, which are turned into associated difference equations by the formal power series in $x$ [66]. For these difference equations we use now the technique described in Ref. [24] and calculate the master integrals for fixed moments $N$, which are inserted into the expression of the unrenormalized OME, see Eq. (4.37) of Ref. [7]. Iteratively, we calculate higher and higher moments. For the complete expression we have calculated 2000 moments and for the contribution with the color factor $O\left(T_{F}^{2} C_{A, F}\right), 8000$ moments. The method of guessing [58,59] can now be used to find a difference equation for the different terms according to their contribution in the Laurent series in $\varepsilon$ and their color and $\zeta$-value factors.

We first have considered the $O(1 / \varepsilon)$ term and extracted the 3-loop anomalous dimension $\gamma_{q g}^{(2)}(N)$ in Ref. [67] solving the difference equations obtained by guessing using the packages Sigma $[48,49]$ EvaluatMultisum and SumProduction [50] and by simplifying the corre-

\footnotetext{
${ }^{1}$ The package uses the codes GiNaC [64] and Fermat [65].
} 
sponding expressions using the package HarmonicSums [51-55]. This is the first independent recalculation of this anomalous dimension since it was first computed in [68].

We turn now to the $O\left(\varepsilon^{0}\right)$ terms. In the case of the $O\left(T_{F}^{2} C_{A, F}\right)$ contributions, the method of guessing has led to difference equations in all cases. The 2000 moments for the remaining color and zeta values were not enough, however, to determine difference equations for the pure color factors and those $\propto \zeta_{3}$, except for the $N_{F}$ terms, for which the number of moments had been sufficient and which also had been calculated before in Ref. [8]. Analyzing the terms with pure color factors and those $\propto \zeta_{3}$ for the contributions $O\left(T_{F}^{2} C_{A, F}\right)$ using Sigma, it turned out that the corresponding difference equations do not factorize in first order completely. Already here we speak of difference equations of order $o=45$ and degree $d \sim 1500$. They could be reduced to low order non-factorizing difference equations. The reason for this is that, as we know through other analyses, elliptic parts are contained in these cases and one expects iterative non-iterative integral solutions here, cf. [30].

For quite a series of color and $\zeta$-factors, namely all but 10 , the constant part of the unrenormalized OME $\hat{A}_{Q g}^{(3)}, a_{Q g}^{(3)}(N)$, could be calculated using the above methods. It is given by

$$
\begin{aligned}
& a_{Q g}^{(3)}(N)=C_{A}^{2} T_{F}\left\{t_{C_{A}^{2} T_{F}}(N)+t_{C_{A}^{2} T_{F} \zeta_{3}}(N) \zeta_{3}\right. \\
& +\frac{72(-2+N)(3+N) P_{2}}{(N-1) N^{2}(1+N)^{2}(2+N)^{2}} \zeta_{4}-\frac{4 P_{17}}{(N-1) N^{2}(1+N)^{2}(2+N)^{2}} \mathrm{~B}_{4} \\
& +p_{q g}^{(0)}\left[-16 \mathrm{~B}_{4} S_{1}+144 \zeta_{4} S_{1}+\left(-16 S_{1}^{3}-\frac{4 P_{7}}{3(N-1) N(1+N)(2+N)} S_{2}-32 S_{1} S_{2}\right.\right. \\
& \left.\left.-8 S_{3}+\left(-\frac{8 P_{8}}{3(N-1) N(1+N)(2+N)}-48 S_{1}\right) S_{-2}-8 S_{-3}+16 S_{-2,1}\right) \zeta_{2}\right] \\
& +\left[\frac{2 P_{35}}{9(N-1)^{2} N^{4}(1+N)^{4}(2+N)^{4}}+\frac{4 P_{31}}{9(N-1)^{2} N^{3}(1+N)^{3}(2+N)^{3}} S_{1}\right. \\
& \left.\left.-\frac{4 P_{18}}{3(N-1) N^{2}(1+N)^{2}(2+N)^{2}} S_{1}^{2}\right] \zeta_{2}\right\}+C_{A} T_{F}^{2}\left\{t_{C_{A} T_{F}^{2}}(N)+t_{C_{A} T_{F}^{2} \zeta_{3}}(N) \zeta_{3}\right. \\
& +N_{F}\left\{-\frac{8 P_{36}}{243(N-1) N^{5}(1+N)^{5}(2+N)^{5}}+p_{q g}^{(0)}\left[\left(\frac{1888}{27} S_{3}+\frac{224}{9} S_{2,1}\right) S_{1}\right.\right. \\
& +\frac{32}{27} S_{1}^{4}+\frac{176}{9} S_{1}^{2} S_{2}+\frac{80}{9} S_{2}^{2}+\frac{640}{9} S_{4}+\left(-\frac{64(2 N-1)}{(N-1) N} S_{1}+\frac{128}{3} S_{2}\right) S_{-2}+\frac{64}{9} S_{-4} \\
& -\frac{32}{3} S_{1,-3}-\frac{64}{3} S_{2,-2}-\frac{32}{9} S_{3,1}+\frac{64(2 N-1)}{(N-1) N} S_{-2,1}+64 S_{1,1,-2}-\frac{416}{9} S_{2,1,1} \\
& \left.+\left(\frac{16}{3} S_{1}^{2}+\frac{16}{3} S_{2}+\frac{32}{3} S_{-2}\right) \zeta_{2}+\left(\frac{448\left(1+N+N^{2}\right)}{9(N-1) N(1+N)(2+N)}-\frac{224}{9} S_{1}\right) \zeta_{3}\right] \\
& +\left(\frac{16 P_{32}}{243(N-1) N^{2}(1+N)^{4}(2+N)^{4}}-\frac{16 P_{10}}{27 N(1+N)^{2}(2+N)^{2}} S_{2}\right) S_{1} \\
& +\frac{8 P_{20}}{81 N(1+N)^{3}(2+N)^{3}} S_{1}^{2}-\frac{16 P_{9}}{81 N(1+N)^{2}(2+N)^{2}} S_{1}^{3}
\end{aligned}
$$




$$
\begin{aligned}
& +\frac{8 P_{26}}{81(N-1) N^{3}(1+N)^{3}(2+N)^{3}} S_{2}-\frac{32 P_{22}}{81(N-1) N^{2}(1+N)^{2}(2+N)^{2}} S_{3} \\
& +\frac{32 P_{21}}{81 N(1+N)^{3}(2+N)^{3}} S_{-2}+\frac{32 P_{15}}{27(N-1) N^{2}(1+N)^{2}(2+N)^{2}} S_{-3} \\
& -\frac{64 P_{16}}{9(N-1) N^{2}(1+N)^{2}(2+N)^{2}} S_{1,-2}-\frac{64 P_{4}}{27 N(1+N)^{2}(2+N)^{2}} S_{2,1} \\
& \left.+\left(-\frac{4 P_{27}}{9(N-1) N^{3}(1+N)^{3}(2+N)^{3}}-\frac{16 P_{5}}{9 N(1+N)^{2}(2+N)^{2}} S_{1}\right) \zeta_{2}\right\} \\
& +\left(-\frac{4 P_{28}}{9(N-1) N^{3}(1+N)^{3}(2+N)^{3}}+\frac{160\left(4-N+N^{2}+4 N^{3}+N^{4}\right)}{9 N(1+N)^{2}(2+N)^{2}} S_{1}\right) \zeta_{2} \\
& \left.+p_{q g}^{(0)}\left(\frac{40}{3} S_{1}^{2}+\frac{40}{3} S_{2}+\frac{80}{3} S_{-2}\right) \zeta_{2}\right\}+C_{F}^{2} T_{F}\left\{t_{C_{F}^{2} T_{F}}(N)+t_{C_{F}^{2} T_{F} \zeta_{3}}(N) \zeta_{3}\right. \\
& -\frac{16(N-1)\left(-2+3 N+3 N^{2}\right)}{N^{2}(1+N)^{2}} \mathrm{~B}_{4}+\frac{72(N-1)\left(-2+3 N+3 N^{2}\right)}{N^{2}(1+N)^{2}} \zeta_{4} \\
& +\left[\frac{P_{23}}{2 N^{4}(1+N)^{4}(2+N)}+\frac{8 P_{19}}{N^{3}(1+N)^{3}(2+N)} S_{1}+\frac{4 P_{3}}{N^{2}(1+N)^{2}(2+N)} S_{1}^{2}\right] \zeta_{2} \\
& +p_{q g}^{(0)}\left[-16 S_{1}^{3}-\frac{8\left(2+3 N+3 N^{2}\right)}{N(1+N)} S_{2}+32 S_{1} S_{2}+16 S_{3}\right. \\
& \left.\left.+\left(-\frac{16}{N(1+N)}+32 S_{1}\right) S_{-2}+16 S_{-3}-32 S_{-2,1}\right] \zeta_{2}\right\}+C_{F}\left\{C _ { A } T _ { F } \left\{t_{C_{F} C_{A} T_{F}}(N)+\right.\right. \\
& t_{C_{F} C_{A} T_{F} \zeta_{3}}(N) \zeta_{3}+\frac{32 P_{13}}{(N-1) N^{2}(1+N)^{2}(2+N)^{2}}\left(\mathrm{~B}_{4}-\frac{9}{2} \zeta_{4}\right) \\
& +p_{q g}^{(0)}\left[32 \mathrm{~B}_{4} S_{1}-144 \zeta_{4} S_{1}+\left(32 S_{1}^{3}-\frac{12 P_{1}}{(N-1) N(1+N)(2+N)} S_{2}-8 S_{3}\right.\right. \\
& \left.\left.+\left(-\frac{8\left(1+3 N+3 N^{2}\right)}{N(1+N)}+16 S_{1}\right) S_{-2}-8 S_{-3}+16 S_{-2,1}\right) \zeta_{2}\right] \\
& +\left(\frac{P_{25}}{18(N-1) N^{3}(1+N)^{3}(2+N)^{3}}-\frac{4 P_{29}}{9(N-1) N^{3}(1+N)^{3}(2+N)^{3}} S_{1}\right. \\
& \left.\left.+\frac{8 P_{12}}{3(N-1) N^{2}(1+N)^{2}(2+N)^{2}} S_{1}^{2}\right) \zeta_{2}\right\}+T_{F}^{2}\left\{t_{C_{F} T_{F}^{2}}(N)+t_{C_{F} T_{F}^{2} \zeta_{3}}(N) \zeta_{3}\right. \\
& +N_{F}\left\{\frac{P_{37}}{243(-1+N) N^{6}(1+N)^{6}(2+N)^{5}}+p_{q g}^{(0)}\left[\left(-\frac{256}{27} S_{3}-\frac{128}{3} S_{2,1}\right) S_{1}\right.\right. \\
& -\frac{32}{27} S_{1}^{4}-\frac{64}{9} S_{1}^{2} S_{2}-\frac{128}{9} S_{2}^{2}+\frac{256}{9} S_{4}-\frac{128}{3} S_{3,1}+\frac{256}{3} S_{2,1,1}-\frac{16}{3} S_{1}^{2} \zeta_{2} \\
& \left.+\left(-\frac{56 P_{14}}{9(-1+N) N^{2}(1+N)^{2}(2+N)}+\frac{224}{9} S_{1}\right) \zeta_{3}\right]+\left(-\frac{16 P_{11}}{243 N^{2}(1+N)^{3}(2+N)}\right.
\end{aligned}
$$




$$
\begin{aligned}
& \left.+\frac{32\left(24+83 N+49 N^{2}+10 N^{3}\right)}{27 N^{2}(1+N)(2+N)} S_{2}\right) S_{1}-\frac{32 P_{6}}{81 N^{2}(1+N)^{2}(2+N)} S_{1}^{2} \\
& +\frac{32\left(24+83 N+49 N^{2}+10 N^{3}\right)}{81 N^{2}(1+N)(2+N)} S_{1}^{3}+\frac{8 P_{33}}{27(-1+N) N^{4}(1+N)^{4}(2+N)^{3}} S_{2} \\
& -\frac{16 P_{24}}{81(-1+N) N^{3}(1+N)^{3}(2+N)^{2}} S_{3}-\frac{128\left(-2-3 N+N^{2}\right)}{3 N^{2}(1+N)(2+N)} S_{2,1} \\
& \left.+\left(\frac{2(-2+N) P_{30}}{9(-1+N) N^{4}(1+N)^{4}(2+N)^{3}}+\frac{16\left(12+28 N+11 N^{2}+5 N^{3}\right)}{9 N^{2}(1+N)(2+N)} S_{1}\right) \zeta_{2}\right\} \\
& +\left(\frac{2 P_{34}}{9(-1+N) N^{4}(1+N)^{4}(2+N)^{3}}+\frac{80\left(6+11 N+4 N^{2}+N^{3}\right)}{9 N^{2}(1+N)(2+N)} S_{1}\right) \zeta_{2} \\
& \left.\left.+p_{q g}^{(0)}\left(-\frac{40}{3} S_{1}^{2}+8 S_{2}\right) \zeta_{2}\right\}\right\}-\frac{64}{9} p_{q g}^{(0)} T_{F}^{3} \zeta_{3} .
\end{aligned}
$$

Here we used the shorthand notation

$$
p_{q g}^{(0)}(N)=\frac{N^{2}+N+2}{(N+2)(N+1) N},
$$

$S_{\vec{a}}$ denote the nested harmonic sums [69]

$$
S_{b, \vec{a}} \equiv S_{b, \vec{a}}(N)=\sum_{k=1}^{N} \frac{(\operatorname{sign}(b))^{k}}{k^{|b|}} S_{\vec{a}}(k), \quad S_{\emptyset}=1, \quad b, a_{i} \in \mathbb{Z} \backslash\{0\},
$$

the constant $B_{4}$ is

$$
\mathrm{B}_{4}=-4 \zeta_{2} \ln ^{2}(2)+\frac{2}{3} \ln ^{4}(2)-\frac{13}{2} \zeta_{4}+16 \mathrm{Li}_{4}\left(\frac{1}{2}\right)
$$

and $P_{i}$ denote polynomials in $N$ (which have be computed explicitly), and the functions $t_{j}(N)$ have still to be calculated. Using the methods of Ref. [24], we have calculated a lot more Feynman diagrams for the color factors contributing to $\hat{\hat{A}}_{Q g}^{(3)}$, which could not yet been gotten using the method of arbitrarily large moments, so that 1122 of 1358 diagrams have been calculated by now.

\section{Conclusions}

Advanced methods in calculating Feynman and master integrals for massive 3-loop OMEs allowed us already to compute a significant part of the contributions to $a_{Q g}^{(3)}$ analytically. Our toolbox employs various methods [24], and relies in particular on very efficient solvers based on difference field and ring theory [39-47]. These algorithms have been implemented in the packages Sigma [48,49], EvaluateMultisums and SumProduction [50]. A very efficient treatment of special functions has been possible using the package HarmonicSums [51-55]. The method of arbitrarily large moments [60] could now be used to calculate the 3-loop anomalous dimension $\gamma_{q g}^{(2)}(N)$ automatically from first principles even in the more involved massive environment [67]. The same method allowed to calculate the contributions to the $O\left(\varepsilon^{0}\right)$ term $a_{Q g}^{(3)}$ for 18 out of 28 of the color- $\zeta$ terms in analytic form. 
Including the use of other methods described in [24], 1122 of 1358 Feynman diagrams contributing to $A_{Q g}^{(3)}$ at $O\left(\varepsilon^{0}\right)$ have been calculated. The remaining terms contain iterative non-iterative integrals over also elliptic letters and are currently being computed.

\section{References}

[1] S. Bethke et al., Workshop on Precision Measurements of $\alpha_{s}$, arXiv:1110.0016 [hep-ph]; S. Moch, S. Weinzierl et al., High precision fundamental constants at the TeV scale, arXiv:1405.4781 [hep-ph];

S. Alekhin, J. Blümlein and S.O. Moch, Mod. Phys. Lett. A 31 (2016) no.25, 1630023.

[2] A. Accardi et al., Eur. Phys. J. C 76 (2016) no.8, 471 [arXiv:1603.08906 [hep-ph]]; S. Alekhin, J. Blümlein, S. Moch and R. Placakyte, Phys. Rev. D 96 (2017) no.1, 014011 [arXiv:1701.05838 [hep-ph]].

[3] S. Alekhin, J. Blümlein, K. Daum, K. Lipka and S. Moch, Phys. Lett. B 720 (2013) 172 [arXiv:1212.2355 [hep-ph]];

A. Gizhko et al., Phys. Lett. B 775 (2017) 233 [arXiv:1705.08863 [hep-ph]].

[4] M. Czakon, P. Fiedler and A. Mitov, Phys. Rev. Lett. 110 (2013) 252004 [arXiv:1303.6254 [hep-ph]].

[5] C. Anastasiou, C. Duhr, F. Dulat, E. Furlan, T. Gehrmann, F. Herzog, A. Lazopoulos and B. Mistlberger, JHEP 1605 (2016) 058 [arXiv:1602.00695 [hep-ph]].

[6] J. Blümlein, A. De Freitas, W.L. van Neerven and S. Klein, Nucl. Phys. B 755 (2006) 272 [hep-ph/0608024].

[7] I. Bierenbaum, J. Blümlein and S. Klein, Nucl. Phys. B 820 (2009) 417 [arXiv:0904.3563 [hep-ph]]; J. Blümlein, S. Klein and B. Tödtli, Phys. Rev. D 80 (2009) 094010 [arXiv:0909.1547 [hep-ph]].

[8] J. Ablinger, J. Blümlein, S. Klein, C. Schneider and F. Wißbrock, Nucl. Phys. B 844 (2011) 26 [arXiv:1008.3347 [hep-ph]].

[9] J. Blümlein, A. Hasselhuhn, S. Klein and C. Schneider, Nucl. Phys. B 866 (2013) 196 [arXiv:1205.4184 [hep-ph]].

[10] A. Behring, I. Bierenbaum, J. Blümlein, A. De Freitas, S. Klein and F. Wißbrock, Eur. Phys. J. C 74 (2014) no.9, 3033 [arXiv:1403.6356 [hep-ph]].

[11] J. Ablinger, A. Behring, J. Blümlein, A. De Freitas, A. Hasselhuhn, A. von Manteuffel, M. Round, C. Schneider, and F. Wißbrock, Nucl. Phys. B 886 (2014) 733 [arXiv:1406.4654 [hep-ph]].

[12] J. Ablinger, A. Behring, J. Blümlein, A. De Freitas, A. von Manteuffel and C. Schneider, Nucl. Phys. B 890 (2014) 48 [arXiv:1409.1135 [hep-ph]].

[13] J. Ablinger, J. Blümlein, A. De Freitas, A. Hasselhuhn, A. von Manteuffel, M. Round and C. Schneider, Nucl. Phys. B 885 (2014) 280 [arXiv:1405.4259 [hep-ph]].

[14] J. Ablinger, J. Blümlein, A. De Freitas, A. Hasselhuhn, A. von Manteuffel, M. Round, C. Schneider and F. Wißbrock, Nucl. Phys. B 882 (2014) 263 [arXiv:1402.0359 [hep-ph]].

[15] J. Ablinger, A. Behring, J. Blümlein, A. De Freitas, A. Hasselhuhn, A. von Manteuffel, C.G. Raab, M. Round, C. Schneider and F. Wißbrock, PoS (EPS-HEP2015) (2015) 504 [arXiv:1602.00583 [hep-ph]].

[16] J. Ablinger et al., DESY 15-112. 
[17] A. Behring, J. Blümlein, A. De Freitas, A. von Manteuffel and C. Schneider, Nucl. Phys. B 897 (2015) 612 [arXiv:1504.08217 [hep-ph]].

[18] A. Behring, J. Blümlein, A. De Freitas, A. Hasselhuhn, A. von Manteuffel and C. Schneider, Phys. Rev. D 92 (2015) no.11, 114005 [arXiv:1508.01449 [hep-ph]].

[19] A. Behring, J. Blümlein, G. Falcioni, A. De Freitas, A. von Manteuffel and C. Schneider, Phys. Rev. D 94 (2016) no.11, 114006 [arXiv:1609.06255 [hep-ph]].

[20] J. Blümlein, A. Hasselhuhn and T. Pfoh, Nucl. Phys. B 881 (2014) 1 [arXiv:1401.4352 [hep-ph]].

[21] J. Blümlein, G. Falcioni and A. De Freitas, Nucl. Phys. B 910 (2016) 568 [arXiv:1605.05541 [hep-ph]].

[22] J. Ablinger, J. Blümlein, A. Hasselhuhn, S. Klein, C. Schneider and F. Wißbrock, Nucl. Phys. B 864 (2012) 52 [arXiv:1206.2252 [hep-ph]].

[23] J. Ablinger, J. Blümlein, C. Raab, C. Schneider and F. Wißbrock, Nucl. Phys. B 885 (2014) 409 [arXiv:1403.1137 [hep-ph]].

[24] J. Ablinger, A. Behring, J. Blümlein, A. De Freitas, A. von Manteuffel and C. Schneider, Comput. Phys. Commun. 202 (2016) 33 [arXiv:1509.08324 [hep-ph]].

[25] J. Ablinger, J. Blümlein, A. De Freitas, A. Hasselhuhn, C. Schneider and F. Wißbrock, Nucl. Phys. B 921 (2017) 585 [arXiv:1705.07030[hep-ph]].

[26] J. Ablinger, J. Blümlein, A. De Freitas, C. Schneider and K. Schönwald, The two-mass contribution to the three-loop pure singlet operator matrix element, arXiv:1711.06717 [hep-ph].

[27] J. Blümlein, A. De Freitas, C. Schneider, and K. Schönwald, DESY 17-187.

[28] J. Ablinger, J. Blümlein, A. De Freitas, A. Goedicke, C. Schneider and K. Schönwald, in preparation.

[29] J. Ablinger et al., DO-TH 17/34.

[30] J. Ablinger, J. Blümlein, A. De Freitas, M. van Hoeij, E. Imamoglu, C. G. Raab, C.-S. Radu and C. Schneider, arXiv:1706.01299 [hep-th].

[31] J. Lagrange, Nouvelles recherches sur la nature et la propagation du son, Miscellanea Taurinensis, t. II, 1760-61; Oeuvres t. I, p. 263;

C.F. Gauß, Theoria attractionis corporum sphaeroidicorum ellipticorum homogeneorum methodo novo tractate, Commentationes societas scientiarum Gottingensis recentiores, Vol III, 1813, Werke Bd. V pp. 5-7;

G. Green, Essay on the Mathematical Theory of Electricity and Magnetism, Nottingham, 1828 [Green Papers, pp. 1-115];

M. Ostrogradski, Mem. Ac. Sci. St. Peters., 6, (1831) 39;

K.G. Chetyrkin and F.V. Tkachov, Nucl. Phys. B 192 (1981) 159.

[32] W.N. Bailey, Generalized Hypergeometric Series, (Cambridge University Press, Cambridge, 1935); L.J. Slater, Generalized Hypergeometric Functions, (Cambridge University Press, Cambridge, 1966); P. Appell and J. Kampé de Fériet, Fonctions Hypergéométriques et Hypersphériques, Polynomes d' Hermite, (Gauthier-Villars, Paris, 1926);

P. Appell, Les Fonctions Hypergéométriques de Plusieur Variables, (Gauthier-Villars, Paris, 1925);

J. Kampé de Fériet, La fonction hypergéométrique, (Gauthier-Villars, Paris, 1937);

H. Exton, Multiple Hypergeometric Functions and Applications, (Ellis Horwood, Chichester, 1976);

H. Exton, Handbook of Hypergeometric Integrals, (Ellis Horwood, Chichester, 1978); 
H.M. Srivastava and P.W. Karlsson, Multiple Gaussian Hypergeometric Series, (Ellis Horwood, Chicester, 1985);

M.J. Schlosser, in: Computer Algebra in Quantum Field Theory: Integration, Summation and Special Functions, C. Schneider, J. Blümlein, Eds., p. 305, (Springer, Wien, 2013) [arXiv:1305.1966 [math.CA]].

[33] E.W. Barnes, Proc. Lond. Math. Soc. (2) 6 (1908) 141; Quart. Journ. Math. 41 (1910) 136; H. Mellin, Math. Ann. 68 (1910) 305;

M. Czakon, Comput. Phys. Commun. 175 (2006) 559 [hep-ph/0511200];

A.V. Smirnov and V.A. Smirnov, Eur. Phys. J. C 62 (2009) 445 [arXiv:0901.0386 [hep-ph]].

[34] F.C.S. Brown, Commun. Math. Phys. 287 (2009) 925 [arXiv:0804.1660 [math.AG]].

[35] F. Wißbrock, $O\left(\alpha_{s}^{3}\right)$ Contributions to the Heavy Flavor Wilson Coefficients of the Structure Function $F_{2}\left(x, Q^{2}\right)$ at $Q^{2} \gg m^{2}, \mathrm{PhD}$ Thesis, TU Dortmund, 2015.

[36] E. Panzer, JHEP 1403 (2014) 071 [arXiv:1401.4361 [hep-th]].

[37] A. von Manteuffel, E. Panzer and R.M. Schabinger, JHEP 1502 (2015) 120 [arXiv:1411.7392 [hep-ph]].

[38] A.V. Kotikov, Phys. Lett. B 254 (1991) 158;

E. Remiddi, Nuovo Cim. A 110 (1997) 1435 [hep-th/9711188];

M. Caffo, H. Czyz, S. Laporta and E. Remiddi, Acta Phys. Polon. B 29 (1998) 2627

[hep-th/9807119]; Nuovo Cim. A 111 (1998) 365 [hep-th/9805118];

T. Gehrmann and E. Remiddi, Nucl. Phys. B 580 (2000) 485 [hep-ph/9912329].

[39] M. Karr, J. ACM 28 (1981) 305.

[40] C. Schneider, RISC, Johannes Kepler University, Linz technical report 01-17 (2001).

[41] C. Schneider, An. Univ. Timisoara Ser. Mat.-Inform. 42 (2004) 163;

J. Differ. Equations Appl. 11 (2005) 799;

Appl. Algebra Engrg. Comm. Comput. 16 (2005) 1.

[42] C. Schneider, J. Algebra Appl. 6 (2007) 415.

[43] C. Schneider, Motives, Quantum Field Theory, and Pseudodifferential Operators (Clay Mathematics Proceedings Vol. 12 ed. A. Carey, D. Ellwood, S. Paycha and S. Rosenberg,(Amer. Math. Soc) (2010), 285 [arXiv:0904.2323].

[44] C. Schneider, Ann. Comb. 14 (2010) 533 [arXiv:0808.2596].

[45] C. Schneider, in: Computer Algebra and Polynomials, Applications of Algebra and Number Theory, J. Gutierrez, J. Schicho, M. Weimann (ed.), Lecture Notes in Computer Science (LNCS) 8942 (2015), 157[arXiv:13077887 [cs.SC]].

[46] C. Schneider, J. Symbolic Comput. 43 (2008) 611 [arXiv:0808.2543v1]; J. Symb. Comput. 72 (2016) 82 [arXiv:1408.2776 [cs.SC]]; J. Symb. Comput. 80 (2017), 616 [arXiv:1603.04285 [cs.SC]].

[47] C. Schneider, Ann. Comb. 9(1) (2005) 75;

S.A. Abramov and M. Petkovšek, J. Symbolic Comput., 45(6) (2010) 684;

C. Schneider, Structural theorems for symbolic summation, Appl. Algebra Engrg. Comm. Comput., 21(1) (2010) 1;

C. Schneider, In: Symbolic and Numeric Algorithms for Scientific Computing (SYNASC), 2014, 15th International Symposium, F. Winkler, V. Negru, T. Ida, T. Jebelean, D. Petcu, S. Watt, D. Zaharie (ed.), (2015) pp. 26; IEEE Computer Society, arXiv:1412.2782v1 [cs.SC]. 
[48] C. Schneider, Sém. Lothar. Combin. 56 (2007) 1, article B56b.

[49] C. Schneider, Computer Algebra in Quantum Field Theory: Integration, Summation and Special Functions Texts and Monographs in Symbolic Computation eds. C. Schneider and J. Blümlein (Springer, Wien, 2013) 325, arXiv:1304.4134 [cs.SC].

[50] J. Ablinger, J. Blümlein, S. Klein and C. Schneider, Nucl. Phys. Proc. Suppl. 205-206 (2010) 110 [arXiv:1006.4797 [math-ph]];

J. Blümlein, A. Hasselhuhn and C. Schneider, PoS (RADCOR 2011) 032 [arXiv:1202.4303 [math-ph]];

C. Schneider, J. Phys. Conf. Ser. 523 (2014) 012037 [arXiv:1310.0160 [cs.SC]].

[51] J. Ablinger, Computer Algebra Algorithms for Special Functions in Particle Physics, Ph.D. Thesis, J. Kepler University Linz, 2012, arXiv:1305.0687 [math-ph].

[52] J. Ablinger, PoS (LL2014) 019; Computer Algebra Algorithms for Special Functions in Particle Physics, Ph.D. Thesis, J. Kepler University Linz, 2012, arXiv:1305.0687 [math-ph];

A Computer Algebra Toolbox for Harmonic Sums Related to Particle Physics, Diploma Thesis, J. Kepler University Linz, 2009, arXiv:1011.1176 [math-ph].

[53] J. Ablinger, J. Blümlein and C. Schneider, J. Math. Phys. 52 (2011) 102301 [arXiv:1105.6063 [math-ph]].

[54] J. Ablinger, J. Blümlein and C. Schneider, J. Math. Phys. 54 (2013) 082301 [arXiv:1302.0378 [math-ph]].

[55] J. Ablinger, J. Blümlein, C.G. Raab and C. Schneider, J. Math. Phys. 55 (2014) 112301 [arXiv:1407.1822 [hep-th]].

[56] G. Almkvist and D. Zeilberger, J. Symb. Comp. 10 (1990) 571; M. Apagodu and D. Zeilberger, Adv. Appl. Math. (Special Regev Issue), 37 (2006) 139.

[57] J. Blümlein, D.J. Broadhurst and J.A.M. Vermaseren, The Multiple Zeta Value Data Mine, Comput. Phys. Commun. 181 (2010) 582 [arXiv:0907.2557 [math-ph]].

[58] M. Kauers, Guessing Handbook, Technical Report RISC 09-07, JKU Linz.

[59] J. Blümlein, M. Kauers, S. Klein and C. Schneider, Comput. Phys. Commun. 180 (2009) 2143 [arXiv:0902.4091 [hep-ph]].

[60] J. Blümlein and C. Schneider, Phys. Lett. B 771 (2017) 31 [arXiv:1701.04614 [hep-ph]].

[61] P. Nogueira, J. Comput. Phys. 105 (1993) 279.

[62] T. van Ritbergen, A.N. Schellekens and J.A.M. Vermaseren, Int. J. Mod. Phys. A 14 (1999) 41 [hep-ph/9802376].

[63] C. Studerus, Comput. Phys. Commun. 181 (2010) 1293 [arXiv:0912.2546 [physics.comp-ph]]; A. von Manteuffel and C. Studerus, arXiv:1201.4330 [hep-ph].

[64] C.W. Bauer, A. Frink and R. Kreckel, Symbolic Computation 33 (2002) 1, cs/0004015 [cs-sc].

[65] R.H. Lewis, Computer Algebra System Fermat, http: / / home bway . net/lewis .

[66] J. Ablinger, A. Behring, J. Blümlein, A. de Freitas and C. Schneider, Algorithms to solve coupled systems of differential equations in terms of power series, arXiv:1608.05376 [cs.SC];

J. Ablinger, J. Blümlein, A. de Freitas, C. Schneider, A toolbox to solve coupled systems of differential and difference equations, In: Proc. of 12th International Symposium on Radiative Corrections (Radcor 2015) and LoopFest XIV (Radiative Corrections for the LHC and Future Colliders), PoS (RADCOR2015) 060, pp. 1-13 [arXiv:1601.01856]. 
[67] J. Ablinger, A. Behring, J. Blümlein, A. De Freitas, A. von Manteuffel and C. Schneider, Nucl. Phys. B 922 (2017) 1 [arXiv:1705.01508 [hep-ph]].

[68] A. Vogt, S. Moch and J.A.M. Vermaseren, Nucl. Phys. B 691 (2004) 129 [hep-ph/0404111].

[69] J.A.M. Vermaseren, Int. J. Mod. Phys. A 14 (1999) 2037 [hep-ph/9806280];

J. Blümlein and S. Kurth, Phys. Rev. D 60 (1999) 014018 [arXiv:hep-ph/9810241]. 